EFECTOS DEL REALCE TEXTUAL Y LA FORMA GRAMATICAL SOBRE LA COMPRENSIÓN Y EL PROCESAMIENTO

José Franco (pp. 30-53)
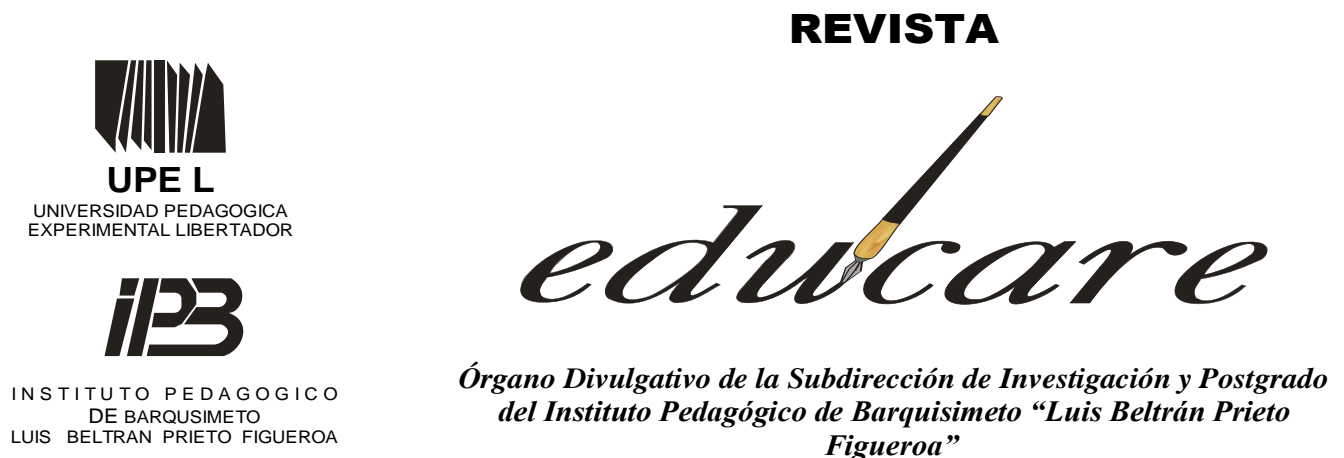

Figueroa"

BARQUISIMETO - EDO. LARA - VENEZUELA

NUEVA ETAPA

FORMATO ELECTRÒNICO

DEPOSITO LEGAL: PPI2O1002LA3674 VOLUMEN 19 № 1

ISSN: 2244-7296

ENERO - ABRIL 2015

\title{
EFECTOS DEL REALCE TEXTUAL Y LA FORMA GRAMATICAL SOBRE LA COMPRENSIÓN Y EL PROCESAMIENTO
}

IMPACT OF TEXTUAL ENHANCEMENT AND TYPE OF LINGUISTIC ITEM

ON EFL LEARNERS' COMPREHENSION AND PROCESSING

\author{
JOSÉ FRANCO* \\ ORANGEL ABREU*
}

"UNIVERSIDAD DE LOS ANDES (ULA) “NúCLEO
UNIVERSITARIO RAFAEL RANGEL" 
EFECTOS DEL REALCE TEXTUAL Y LA FORMA GRAMATICAL SOBRE LA COMPRENSIÓN Y EL PROCESAMIENTO

José Franco (pp. 30-53)

\section{EFECTOS DEL REALCE TEXTUAL Y LA FORMA GRAMATICAL SOBRE LA COMPRENSIÓN Y EL PROCESAMIENTO \\ IMPACT OF TEXTUAL ENHANCEMENT AND TYPE OF LINGUISTIC ITEM ON EFL LEARNERS' COMPREHENSION AND PROCESSING}

\begin{tabular}{|c|c|}
\hline TRABAJO DE INVESTIGACIÒN & $\begin{array}{c}\text { José Franco* } \\
\text { Orangel Abreu }^{* *} \\
\text { ULA }\end{array}$ \\
\hline Recibido:22/11/2014 & Aceptado:29/03/2015 \\
\hline RESUMEN & ABSTRACT \\
\hline $\begin{array}{l}\text { El presente estudio exploró los efectos del } \\
\text { realce textual en la modalidad de letras } \\
\text { mayúsculas y el tipo de forma gramatical } \\
\text { (forma pasiva y presente perfecto) sobre la } \\
\text { comprensión y el procesamiento en ochenta } \\
\text { estudiantes universitarios de Inglés como } \\
\text { Lengua Extranjera (ILE) del nivel I } \\
\text { pertenecientes a dos instituciones universitarias } \\
\text { distintas: Universidad del Zulia y Universidad } \\
\text { Pedagógica Experimental Libertador, Instituto } \\
\text { Pedagógico de Barquisimeto "Luis Beltrán } \\
\text { Prieto Figueroa". Un Análisis Factorial Múltiple } \\
\text { de Varianza (MANOVA) reveló que el realce } \\
\text { textual no tuvo un impacto significativo en la } \\
\text { comprensión y/o el procesamiento de los } \\
\text { participantes, caso contrario a la forma } \\
\text { gramatical. Los resultados conllevaron a } \\
\text { elaborar un Análisis Factorial de Varianza } \\
\text { (ANOVA) para la comprensión y el } \\
\text { procesamiento. Los análisis mostraron que la } \\
\text { forma pasiva resultó más beneficiosa en } \\
\text { términos de comprensión, mientras que el } \\
\text { presente perfecto mostró ser más efectivo para } \\
\text { el procesamiento. Se concluye que los } \\
\text { profesores de inglés deben diseñar actividades } \\
\text { de clase y tareas que se adapten al grado de } \\
\text { dificultad de tales formas gramaticales para } \\
\text { promover su procesamiento más efectivamente. } \\
\text { Descriptores: realce textual, forma gramatical, } \\
\text { comprensión. }\end{array}$ & $\begin{array}{l}\text { The purpose of this research was to study the } \\
\text { impact of textual enhancement (capital letters) } \\
\text { and type of linguistic item (passive voice \& } \\
\text { present perfect) on an EFL class (beginners) } \\
\text { from two Venezuelan universities: Universidad } \\
\text { del Zulia and Universidad Pedagógica } \\
\text { Experimental Libertador - Barquisimeto. First, } \\
\text { a MANOVA showed textual enhancement had a } \\
\text { low impact on comprehension and processing in } \\
\text { contrast to type of linguistic item whose impact } \\
\text { was high. These results led the researchers to } \\
\text { apply an ANOVA for comprehension and } \\
\text { processing. This time results revealed passive } \\
\text { voice is more beneficial regarding } \\
\text { comprehension, whereas present perfect was } \\
\text { more effective for processing. It is concluded } \\
\text { that English teachers should design classroom } \\
\text { activities and tasks to suit the degree of } \\
\text { difficulty of such grammatical forms to promote } \\
\text { processing more effectively. }\end{array}$ \\
\hline
\end{tabular}

\footnotetext{
* Egresado de la Universidad de los Andes, Núcleo Universitario "Rafael Rangel". Actualmente docente investigador adscrito al Departamento de Lenguas Modernas de la misma universidad y miembro del Grupo de Investigación en Lenguas Extranjeras (GILE). Francojo@ula.ve.

** Egresado de la Universidad del Zulia. Actualmente docente investigador adscrito al Departamento de Lenguas Modernas del Núcleo Universitario "Rafael Rangel” y miembro del Grupo de Investigación en Lenguas Extranjeras (GILE). O.abreu@ula.ve
} 


\section{INTRODUCCIÓN}

Los años 80 trajo consigo ciertos cambios de preferencia en lo referente a los métodos instruccionales de los idiomas al generalizarse un rechazo hacia la enseñanza de una Segunda Lengua (SL) o una Lengua Extranjera (LE) basada únicamente en la forma (e.g., "Grammar Translation Method", "Audiolingual Method", "Total Physical Response”), esto a su vez provocó que los profesores de idiomas adoptaran pedagogías basadas en el significado (e.g., "Communicative Teaching", "Immersion Instruction", "The Natural Approach", "Content-Based Instruction") (Leow, 2001; Raimes, 1991). En consecuencia, éstos se han visto en la necesidad de proseguir los objetivos de la competencia comunicativa y la fluidez, mientras que la enseñanza de la gramática que no contribuye a las metas comunicativas ha sido considerada como contraproducente. Sin embargo, los años 90 trajo algunas dudas acerca de la validez de las propuestas de enseñanza que se enfocaban principalmente en el significado.

Estas inquietudes han derivado principalmente de estudios acerca de la situación de inmersión en francés de la Canadá anglófona (Day y Shapson, 1991; Hammerly, 1987; Harley, 1993; Harley y Swain, 1985; Swain, 1985; Swain y Lapkin, 1989, citados en Lee, 2007), los cuales desde mediados de 1980 vienen reportando la dificultad de los estudiantes con el uso preciso de la SL, aún después de ser sometidos a un periodo sustancial de estudio en un primer programa basado en el significado. Tal observación ha conducido a los investigadores que estudian la adquisición de una segunda lengua a investigar cómo integrar eficientemente la enseñanza de la gramática, sin obstaculizar los objetivos de la instrucción basada en el significado.

Una opción que ha tenido un impacto significativo en la agenda relacionada con la atención del estudiante es la teoría del enfoque en la forma, una propuesta para la enseñanza de la gramática en la cual la atención es atraída brevemente hacia una forma lingüística en un contexto significativo (Long, 1991; Long y Robinson, 1998, citado en Lee, ob. cit). Uno de los postulados fundamentales de este enfoque es que la atención del estudiante puede ser dirigida al aprendizaje tanto de la forma como también del contenido. 
Es a partir de esta teoría que algunos investigadores se han abocado a indagar más este campo, siendo algunos de éstos Leow (1997, 2001), Leow, Egi, Nuevo y Tsai (2003), Lee (2007), Overstreet (1998, citado en Wong, 2003), Lee (2007), Wong (2003), De Santis (2008) y Simard (2009) quienes examinaron los efectos del realce textual como un punto óptimo de intervención para alcanzar el objetivo del enfoque en la forma. Sus argumentos se basan en tres puntos claves: (a) La relevancia que posee el rol de la atención al promover el procesamiento de la información gramatical contenida en el input, (b) la condición del realce textual como una técnica menos obstruccionista al atraer la atención de los estudiantes hacia la forma en comparación con otras técnicas más explícitas, tales como: la instrucción gramatical, el feedback, entre otras (Doughty y Williams, 1998; Sharwood Smith, 1981; White, 1998, citado en Lee, 2007) y (c) dos estudios (Shook, 1994 y Jourdenais, Ota, Stauffer, Boyson y Doughty, 1995; citados en Leow, 1997) cuyos resultados mostraron hallazgos positivos para dicha técnica.

Asimismo, existe un creciente número de estudios que han examinado los efectos del realce textual junto con otras variables intervinientes, tales como direcciones explicitas para prestar atención a la forma (Shook, 1994, citado el Leow, 1997; Dalili, Ketabi, Kassaian y Rasekh, 2011; Nahavandi, Mukundan, 2013; Aghajami, Rahimy, 2013), longitud textual (Leow, ob. cit), familiaridad con el tópico (Overstreet, 1998, citado en Wong, 2003 y Lee, 2007), enfatización de las formas (Leow et al., 2003), input simplificado (Wong, 2003) y las tareas de producción (Izumi, 2002).

Respondiendo a la necesidad de aportar nuevas experiencias en el campo de la adquisición de una segunda lengua, esta investigación, integró tres elementos determinantes:

1) Las letras mayúsculas como una modalidad de realce textual que ha resultado beneficiosa en términos de procesamiento (Shook, 1994; citado en Leow, ob. cit y Simard, 2009) y que, sin embargo, no existe evidencia de que la misma resulte beneficiosa en términos de comprensión.

2) Dos formas gramaticales de impacto visual considerable poco usadas en investigaciones relacionadas con el realce textual, son: a) la forma pasiva del inglés que ha 
sido empleada solamente en Lee (ob. cit) y b) el presente perfecto del inglés, que según la revisión de literatura no ha sido empleada en anteriores investigaciones.

3) Dos instituciones universitarias diferentes (Universidad del Zulia (LUZ) y Universidad Pedagógica Experimental Libertador, Instituto Pedagógico “Luis Beltrán Prieto Figueroa" de Barquisimeto (UPEL-IPB).

En relación a este último aspecto, algunas investigaciones disponibles que han empleado estudiantes pertenecientes a instituciones diferentes (Wong, ob. cit; Lee, ob. cit y Simard, ob.cit) no establecen de manera explícita comparaciones intergrupales, lo cual, tal como ya se ha mencionado, representa un aspecto importante a ser evaluado en el presente trabajo.

Tomando como base todo lo antes descrito, esta investigación se planteó cuatro propósitos fundamentales: 1) establecer las posibles diferencias entre las instituciones en términos de comprensión y procesamiento, 2) examinar los posibles efectos del realce textual sobre la comprensión y el procesamiento, 3) verificar los posibles efectos de las formas gramaticales sobre la comprensión y el procesamiento y 4) evaluar la posible interacción entre el realce textual y la forma gramatical.

\section{FUNDAMENTACIÓN TEÓRICA}

La experiencia de inmersión en Francés de la Canadá anglófona (Day y Shapson, 1991; Hammerly, 1987; Harley, 1993; Harley y Swain, 1985; Swain, 1985; Swain y Lapkin, 1989, citados en Lee, ob. cit), nos muestra que la instrucción puramente comunicativa o lo que Long (1991) y Long y Robinson (1998, citado en Lee, ob. cit) llaman "enfoque en el significado", no garantiza el aprendizaje de una SL o LE. Ellos argumentan que la instrucción basada en la forma y la instrucción basada en el contenido son igualmente valiosas y que deben complementarse en lugar de excluirse.

Long (1991) y Long y Robinson (1998, citado en Lee, ob. cit) a lo largo de sus investigaciones, han formulado una teoría denominada "Teoría del Enfoque en la Forma". Esta teoría se basa fundamentalmente en un tipo de instrucción que, por un lado, presenta la importancia de los principios comunicativos de la enseñanza de una lengua, y, por el otro, 
justifica la necesidad de dirigir la atención a formas gramaticales de dificultad considerable presentes en la lengua meta, tales como aquellas que no tienen valor semántico inherente, y que por lo tanto, no contribuyen al significado referencial de una oración o expresión (Bransdorfer, 1991 y VanPatten, 1996, 2000 citados en Wong, ob. cit).

Es imprescindible entender la importancia de la atención en el procesamiento del input. De acuerdo con Leow (ob. cit) el rol de la atención al promover procesamiento subsiguiente de información gramatical contenida en el input ha sido generalmente aceptado por muchas áreas de investigación, incluyendo la ciencia cognitiva (Tomlin y Villa, 1994; citado en Leow, ob. cit), la psicología cognitiva (Carr y Curran, 1994; citado en Leow, ob. cit) y el campo investigativo de la adquisición de una SL (Robinson, 1995 y Schmidt, 1990; citado en Leow, ob. cit). Asimismo, señala que la manera cómo es atraída la atención de los estudiantes en los diferentes estudios que se han realizado puede implicar desde la utilización de técnicas implícitas o incidentales (e.g., realce textual) hasta técnicas explicitas (e.g. instrucción explícita o feedback).

\section{El Realce Textual}

El realce textual según Lee (ob. cit), es una técnica implícita del enfoque en la forma que utiliza métodos de realce visual, tales como: subrayado, negrillas, combinación de colores, itálicas, mayúsculas o tipos de letra diferentes. Tal técnica ha sido investigada como un medio para promover el procesamiento de ítems lingüísticos en un gran número de estudios.

Aún cuando existe un número considerable de estudios relacionados con el realce textual como un vehículo óptimo para atraer la atención de los estudiantes hacia algunas formas o aspectos gramaticales presentes en el input, esta revisión de literatura puso de manifiesto una serie de contradicciones en los resultados obtenidos en los diferentes estudios relacionados con dicha técnica. Mientras que unos estudios muestran hallazgos positivos para el realce textual (e.g. Shook, 1994 y Jourdenais y otros 1995 citados en Leow, ob. cit, Lee, ob. cit; De Santis, 2008; Simard, ob. cit; Aghajani y Rahimy, 2013; Nahavandi y Mukundan, 2013; Jabbarpoor y Abdollahzadeh, 2013 y Ayiewbey, 2013), otras investigaciones, como en el caso de Leow (ob. cit), Overstreet (1998, citado en Wong, 
ob. cit), Leow (ob. cit), Wong (ob. cit), Leow, Egi, Nuevo y Tsai (2003), Dalili, Ketabi, Kassaian y Rasekh (2011) y Sarkhosh (2012) han encontrado que dicha técnica no presenta efectos beneficiosos. En otras palabras, contrario a lo que podría esperarse, los resultados de algunos estudios sugieren que los estudiantes independientemente del realce textual, en algunos casos comprendieron o procesaron de manera exitosa el input al cual fueron expuestos.

\section{El Realce Textual y el Formato o Modalidad}

En lo que respecta al primer aspecto, es importante mencionar que no existe una línea investigativa que haya comparado los efectos de las diferentes modalidades de realce sobre el procesamiento, sin embargo, Shook (1994, citado en Leow, 1997) al examinar los efectos de la modalidad o tipo de realce textual sobre el procesamiento de dos formas gramaticales distintas (el presente perfecto y los pronombres relativos quelquien del español) encontró que el realce textual en la modalidad de letras mayúsculas fue beneficioso para el procesamiento.

Atendiendo lo antes planteado, si observamos la cantidad de modalidades comparadas, encontramos que la evidencia continua siendo exigua. En una investigación Simard (ob. cit) establece una comparación más profunda al explorar los efectos de ciertas modalidades del realce textual sobre el procesamiento de las terminaciones del plural en inglés. En su estudio participaron 188 estudiantes hablantes nativos de francés, pertenecientes al primer año de secundaria de diferentes institutos de Quebec, Canadá. Los participantes fueron sometidos a ocho condiciones diferentes: 1) Terminaciones realzadas con itálicas, 2) terminaciones realzadas con subrayado, 3) terminaciones realzadas con negrillas, 4) terminaciones realzadas con color amarillo, 5) terminaciones realzadas con letras mayúsculas, 6) terminaciones realzadas combinando todas las modalidades anteriores, 7) terminaciones realzadas combinando negrillas, mayúsculas y subrayado y 8) terminaciones sin realzar (grupo control).

Los resultados mostraron que el realce textual en la modalidad de letras mayúsculas fue más beneficioso para atraer la atención de los aprendices hacia la forma con respecto a 
las demás modalidades, lo cual fue considerado dentro de las decisiones metodológicas de la siguiente investigación.

Sarkhosh (ob. cit) exploró los efectos del realce textual al emplear el subrayado, negrillas y cursivas. Los resultados mostraron que el subrayado y las negrillas tuvieron mucha más efectividad que las cursivas al fomentar el procesamiento del pasado simple del inglés. Por su parte, Ayiewbey (2013) encontró resultados similares a los descritos anteriormente. El realce textual en la modalidad de negrillas resultó ser más efectivo que las cursivas en el aprendizaje del pasado simple del inglés. Su trabajo es una réplica parcial de Sarkhosh (ob. cit).

Ambos autores concluyen sugiriendo que los tipos de formato que se emplearon para resaltar las estructuras gramaticales puede revestir tanta importancia como el propio formato que pueda emplear el aprendiz, ya que este se adapta a su propio estilo y estrategia de aprendizaje. Con ello se constata la importancia de dicha técnica y la necesidad de explorar con otros tipos de modalidades.

\section{El Realce Textual y otras Variables Intervinientes}

Los estudios que han examinado los efectos del realce textual junto con otras variables pudieran igualmente, aportar elementos importantes para estas investigaciones. Leow (ob. cit), al examinar los efectos del realce y la longitud textual sobre la comprensión y el procesamiento del imperativo impersonal del español halló un rol positivo para la longitud textual sobre la comprensión. Sin embargo, el realce textual no tuvo efectos significativos ni en la comprensión, ni en el procesamiento.

Asimismo, Overstreet (1998, citado en Wong, ob. cit) motivado por la hipótesis de que la lectura de textos familiares reduciría el esfuerzo cognitivo, evaluó los efectos del realce textual y la familiaridad del contenido sobre la comprensión del pretérito y el imperfecto del español. Los resultados no le atribuyeron efectos positivos al realce textual, ni a la familiaridad del tópico con respecto a la comprensión.

Lee (ob. cit), sin embargo, al emplear las mismas variables de Overstreet (1998) encontró que el realce textual resultó beneficioso para el aprendizaje de la forma pasiva del inglés, pero no para la comprensión del significado. La familiaridad con el tópico por el 
contrario, favoreció la comprensión del significado, pero no fue efectiva en el aprendizaje de la forma.

Leow (ob. cit) hizo una réplica parcial de su trabajo presentado en 1997. Utilizó la misma forma gramatical (el imperativo en español), sin embargo, añadió una tarea más, siendo ésta, la producción escrita. Los resultados fueron muy parecidos a los de su trabajo anterior, no hubo diferencias significativas del realce textual con respecto a los textos no realzados, ni efectos positivos de dicha técnica sobre la comprensión, el procesamiento y la producción escrita.

Wong (ob. cit) estudió los efectos del realce textual y el input simplificado sobre la adquisición de las cláusulas relativas en pasado en aprendices de francés como SL. Por medio de los resultados se logró constatar que no hubo efectos estadísticamente significativos del realce textual con respecto a la adquisición.

Dalili, Ketabi, Kassaian y Rasekh (ob. cit) al comparar los efectos del realce textual y el aprendizaje sistemático, concluyeron que este último resultó más beneficioso en el aprendizaje de la alternación dativa del inglés.

Los estudios citados anteriormente evidencian la necesidad de explorar los efectos del realce textual con otras variables intervinientes igualmente importantes, ya que están ligadas a procesos cognitivos esenciales en el aprendizaje una SL o LE. A su vez, es un indicativo de que es un campo que amerita ser investigado más a fondo.

\section{El Realce Textual y la Forma Gramatical}

Jensen y Vinther (2003) definen la forma gramatical como las características formales de la lengua bien sean escritas o habladas presentes en el input.

A excepción de Shook (1994, 1999), no muchos estudios han examinado cómo este tipo de realce del input podría impactar la adquisición de las formas que no tienen valor comunicativo, es decir, formas que no tienen valor semántico inherente, por lo tanto, no contribuyen al significado referencial de una oración o expresión (Bransdorfer, 1991; VanPatten, 1996, 2000; citados en Wong, ob. cit). Según el modelo de procesamiento de VanPatten (VanPatten, 1996, 2000; citados en Wong, ob. cit) estas formas son más difíciles de adquirir. Ocurre porque los aprendices poseen una capacidad limitada para procesar el 
input y también porque éstos, tienden a tratar de entender el contenido del mensaje antes de prestar atención a cómo ese mensaje es codificado lingüísticamente. Ellos prestarán atención a las formas que contengan más significado en el input antes que a las menos significativas, es decir, aquellas que tienen menos valor comunicativo.

La cuestión de qué formas realzar (o no realzar) es un asunto complejo y la evidencia existente es exigua pero sugestiva, no obstante lo planteado anteriormente nos permite hasta cierto punto discriminar el grado de complejidad de una forma gramatical en gran medida por su valor comunicativo. Han, Park y Combs (2008) proponen que las formas gramaticales poseen diferencias inter-estructurales que vienen dadas por algunos aspectos, tales como: el valor comunicativo, la complejidad formal, la complejidad funcional, la carga semántica, la carga perceptiva y las reglas subyacentes.

Jabbarpoor y Abdollahzadeh (2013) argumentan que las formas morfológicamente más simples tienen más posibilidad de ser procesadas exitosamente, mientras que las formas sintácticas más complejas requieren procesamiento más profundo y secuencial.

Simard (ob. cit) expresa que muchas de las formas lingüísticas empleadas en estudios relacionados con el realce textual contienen algún grado de valor semántico (e.g., las formas y los tiempos verbales) y en la medida en que este valor presente variaciones, así mismo pudieran variar el grado de comprensión y/o procesamiento.

Shook (1994, citado en Leow, 1 ob. cit) al examinar los efectos de la modalidad o formato del realce textual sobre el procesamiento de dos formas gramaticales distintas (el presente perfecto y los pronombres relativos quelquien del español) encontró que el realce textual fue beneficioso para el procesamiento. Adicionalmente, los participantes en este estudio procesaron en mayor grado el presente perfecto que los pronombres relativos del español. Esto evidencia que las formas gramaticales que contienen poco valor comunicativo pueden resultar procesadas en menor grado en comparación con aquellas que poseen un alto valor comunicativo.

Posteriormente, Jourdenais y otros (1995, citados en Leow, ob. cit), al igual que Shook (1994, citado en Leow, ob. cit) encontraron que el realce textual tuvo un efecto positivo al promover el procesamiento subsiguiente del pretérito y el imperfecto del español. Atendiendo a estos hallazgos, se puede notar que las formas gramaticales 
utilizadas en este estudio, no varían estructuralmente una de la otra, ya que ambas poseen solamente un morfema. No obstante, el valor comunicativo de éstas no es claro.

Leow (ob. cit) encontró que el realce textual no tuvo efectos significativos sobre la comprensión y el procesamiento del imperativo formal del español. En una investigación más reciente, Leow y otros (2003) incluyeron dos tipos de formas gramaticales distintos a los de Leow (2001), éstas fueron el presente perfecto y el presente subjuntivo del español. Los resultados no indicaron efectos significativos con respecto a la comprensión o al procesamiento.

Un estudio que resulta importante en esta revisión de literatura concerniente al realce textual y las formas gramaticales es el de Jabbarpoor y Abdollahzadeh (ob. cit), en el cual incluyeron cuatro formas gramaticales del inglés (preposiciones, verbos modales, modo subjuntivo y estructuras de inversión o alternación dativa). Los resultados además de mostrar la efectividad del realce textual, evidenciaron que el modo subjuntivo y las estructuras de inversión del inglés resultaron más beneficiosos para el aprendizaje.

Lo expuesto anteriormente, evidencia que existe la posibilidad de investigar acerca de qué formas gramaticales se deben realzar (o no realzar). VanPatten (1996) sostiene que las formas gramaticales pueden dividirse en tres categorías: a) de valor comunicativo alto (e.g., la forma -ing del inglés) b) de valor comunicativo medio y c) de valor comunicativo bajo (e.g., la terminación $-s$ de la tercera persona del singular en inglés). Estas categorías se basan en el valor semántico de las palabras así como también en su redundancia estructural. De Santis (2008) encontró efectos significativos en el realce textual con respecto a la identificación de la inflexión verbal -s de la tercera persona del singular en presente en inglés.

Las formas lingüísticas que han sido tomadas para los estudios revisados, pertenecen en su mayoría al inglés y al español. Los hallazgos de Shook (1994, citado en Leow, ob. cit) y Simard (ob. cit), dejan ver que el realce textual en la modalidad de mayúsculas es más efectivo en comparación con otras modalidades, ya que, en los dos estudios que ha sido utilizada, los hallazgos han sido positivos.

Considerando los hallazgos de Shook (1994, citado en Leow, ob. cit) al emplear el presente perfecto en español para su estudio, un aspecto innovador para ser utilizado en esta 
investigación sería estudiar si dicha forma gramatical en inglés pudiera tener un efecto equivalente. Lee (ob. cit), por su lado, empleó la forma pasiva en su estudio, logrando hallazgos positivos para el realce textual, sin embargo, cabría preguntarse si la misma es procesada de igual manera por estudiantes cuya lengua nativa es el español.

Tal como puede notarse a partir de le revisión de literatura hecha, las decisiones metodológicas en cuanto a modalidad de realce textual y forma gramatical varían considerablemente. De igual manera, resulta interesante la cantidad de variables intervinientes que se han explorado con el realce textual. Sin embargo, a fin de delimitar la metodología de este estudio, se integran tres factores determinantes, por un lado, las letras mayúsculas como una modalidad de realce textual que ha resultado beneficiosa en términos de procesamiento (Shook, 1994; citado en Leow, ob. cit y Simard, ob. cit). Un elemento crucial en este estudio fue verificar si la misma resulta beneficiosa en términos de comprensión.

Por otro lado, dos formas gramaticales de impacto visual considerable poco usadas en investigaciones relacionadas con el realce textual, éstas son: a) la forma pasiva del inglés que ha sido empleada solamente en Lee (2007) y b) el presente perfecto del inglés, que según la revisión de literatura no ha sido empleada en anteriores investigaciones.

\section{El Realce Textual, la Comprensión y el Procesamiento}

La comprensión según Richards, Platt y Weber (1985) es el proceso mediante el cual una persona entiende el significado de la lengua escrita o hablada. En la revisión de literatura disponible se comprobó que, por un lado, en diversas investigaciones relacionadas con el realce textual se ha incluido la comprensión (Overstreet, 1998 citado en Wong, ob. cit; Leow, ob. cit), mientras que por el otro, es bastante evidente la inclusión del procesamiento (Shook, 1994 citado en Leow, ob. cit; Simard, ob. cit; Sarkhosh, ob. cit y Jourdenais y otros, 1995 citados en Leow, ob. cit), el cual es definido según Leow (ob. cit) como: "esa parte del input que ha sido atendida por los aprendices mientras procesan el input. El procesamiento representa los datos lingüísticos que pueden ser usados para reconocimiento inmediato y no implica necesariamente la adquisición de la lengua" (p. $334)$. 
De suma importancia resulta el hecho de que algunos autores han explorado los efectos del realce textual sobre la comprensión y el procesamiento de manera simultánea (Leow, ob. cit, y Lee, ob. cit), lo cual es un aspecto que se incluye en el presente estudio.

\section{METODOLOGÍA}

De acuerdo al problema que se ha planteado, esta investigación es de tipo experimental. Esta consiste en la aplicación de una serie de tareas que deben ser realizadas por los estudiantes participantes en la investigación (Hernández, Fernández y Baptista, 1998). Estas tareas o instrumentos de recolección son la prueba de comprensión y la prueba de procesamiento. Se aplicó un diseño de tipo experimental. Este diseño se realizó bajo condiciones naturales de los sujetos estudiados y tuvo una duración de una sesión por grupo.

\section{Participantes}

En la presente investigación participaron un total de 80 estudiantes, de los cuales 41 pertenecían a LUZ y 39 a la UPEL-IPB. Todos pertenecientes al primer nivel de inglés como lengua extranjera. Dichos participantes fueron en su mayoría del género femenino (51 mujeres y 29 hombres) y con edades comprendidas entre 16 y 40 años ( $M=20,25$, DT= 4,67). Se eligieron estas dos instituciones a fin de obtener una muestra importante de la cual pudieran derivar resultados lo suficientemente confiables para poder generalizar las consideraciones respectivas que derivan de este estudio.

\section{Instrumentos}

Se utilizaron dos textos, uno para la forma pasiva y otro para el presente perfecto. El primer texto se titula The Spirit of the Dead (El Espíritu de los Muertos), es la versión original utilizada por Lee (2007). Este contiene 12 expresiones en forma pasiva que han sido realzadas (en letras mayúsculas) para el grupo experimental. La versión para el grupo control no contendrá realce textual a fines de establecer las comparaciones respectivas para ambos grupos. 
El segundo texto, se titula Agroecology ${ }^{\dagger}$ (Agroecología), este es una versión adaptada de un método de enseñanza de inglés como LE y contiene 11 expresiones en presente perfecto, de igual manera realzadas (en letras mayúsculas) para el grupo experimental y sin realce para el grupo control.

Para la recolección de datos se empleó una tarea de comprensión y otra de procesamiento. La primera consistió en responder una serie de preguntas de comprensión (11 para la forma pasiva y 10 para el presente perfecto) relacionadas directamente con el texto. Esta tarea fue empleada originalmente por Leow (ob. cit) y fue adaptada para esta investigación a las formas gramaticales empleadas (la forma pasiva y el presente perfecto).

La segunda tarea, o de procesamiento, consistió en 11 ítems relacionados exclusivamente con las formas gramaticales empleadas. En cada ítem, los participantes seleccionaron una de cuatro posibles respuestas. Fue empleada por Leow (ob. cit) y adaptada para este estudio a las formas gramaticales (forma pasiva y presente perfecto).

\section{Procedimiento}

A fin de evitar la intervención de cualquier factor en la recolección de la información (e.g. conocimiento previo), se procedió inicialmente a investigar por medio de los profesores de los cursos si las formas gramaticales que fueron empleadas en este estudio habían sido tratadas en clase, constatándose que no. Posteriormente, los participantes de cada institución fueron divididos en grupos relativamente consistentes en número. Para ello fue utilizada una tabla de selección al azar. Cada individuo tuvo la misma probabilidad de ser insertado en una de las cuatro condiciones que a continuación se presentan:

(a) un texto realzado en forma pasiva

(b) un texto sin realzar en forma pasiva

(c) un texto realzado en presente perfecto y

(d) un texto sin realzar en presente perfecto.

Los participantes referidos a la condición de realce textual, conformaron el grupo experimental, mientras que aquellos no considerados a esta condición, conformaron el

\footnotetext{
${ }^{\dagger}$ Versión adaptada de www.cnr.berkeley.edu/\%7Eagroeco3/the_potential_of_agroecology.html
} 
grupo control.

Cada grupo recibió una de las versiones de los textos The Spirit of the Dead (El Espíritu de los Muertos) para la forma pasiva y Agroecology (Agroecología) para el presente perfecto. Antes de comenzar a desarrollar las tareas, se pidió a los estudiantes que mientras desarrollaran las mismas, no volvieran a las secciones anteriores para verificar las respuestas, esto a fin de evitar interferencias en la medición de los resultados de las pruebas de comprensión y procesamiento. Luego de leer el texto, los participantes llevaron a cabo la tarea de comprensión y posteriormente a esta, la de procesamiento. Cabe destacar que cada sesión por grupo tuvo una duración de 18 minutos que se distribuyeron de la siguiente manera:

8 minutos para la lectura del texto

5 minutos para desarrollar la tarea de comprensión y

5 minutos para desarrollar la tarea de selección múltiple.

La distribución de tiempo fue basada en la establecida por Wong (ob. cit), así como también en las longitudes de los textos (315 palabras para la forma pasiva y 248 para el presente perfecto). Asimismo, se consideró la dificultad de las tareas de comprensión y procesamiento, las cuales, según Leow (ob. cit) no son de naturaleza compleja.

Cabe destacar, sin embargo, que dichas tareas derivan directamente de la lectura por lo cual se les suministró una mayor cantidad de tiempo en comparación con las demás actividades.

\section{Análisis de los Datos}

El análisis de los resultados de este estudio se hizo a través de un Análisis Factorial Múltiple de Varianza (MANOVA) utilizando el Paquete Estadístico para las Ciencias Sociales (SPSS). En primer lugar, a través una prueba $t$ se pudo obtener las estadísticas descriptivas de cada uno de los grupos participantes en el estudio. Se calcularon sus respectivas medias de comprensión y procesamiento para luego ser comparadas.

Seguidamente y para dar respuesta a la segunda pregunta de la investigación se elaboró un Análisis Factorial Múltiple de Varianza para verificar los efectos del realce 
EFECTOS DEL REALCE TEXTUAL Y LA FORMA GRAMATICAL SOBRE LA COMPRENSIÓN Y EL PROCESAMIENTO

José Franco (pp. 30-53)

textual, la forma gramatical y su interacción. Posteriormente, se realizó un Análisis de Varianza Simple (ANOVA) para cada variable dependiente para establecer las diferencias que pudiera tener cada forma gramatical en términos de comprensión y procesamiento.

\section{RESULTADOS}

La presente sección corresponde a la presentación de los resultados obtenidos. Primeramente, se presentan los resultados de la prueba $t$, la cual se aplicó con el fin de examinar la heterogeneidad u homogeneidad de los grupos (UPEL y LUZ) en términos de comprensión y procesamiento, para así llevar a cabo los análisis por institución o como una sola muestra. En segundo lugar, y de acuerdo a los objetivos propuestos, se presenta un Análisis Factorial Múltiple de Varianza donde se analizan las variables: realce textual, forma gramatical y su respectiva interacción. Posteriormente, se presentan los resultados arrojados por los Análisis de Varianza Simple para las variables dependientes con sus respectivas medias y desviaciones estándar.

\section{Efectos del Realce Textual y la Forma Gramatical}

Los resultados indicaron que sólo hubo un efecto estadísticamente significativo para la forma gramatical $[F(2,75)=12,465, p=0,00]$, es decir, que independientemente del realce textual, una de las dos formas gramaticales fue comprendida o procesada en mayor grado. Estos resultados se muestran en la tabla 1.

Tabla 1. Análisis Factorial Múltiple de Varianza.

\begin{tabular}{lcccccc}
\hline Fuente & Valor & $\begin{array}{c}\text { Grados de } \\
\text { Libertad }\end{array}$ & $\begin{array}{c}\text { Grados de } \\
\text { Libertad del } \\
\text { Error }\end{array}$ & F & p & $*$ * \\
\hline Realce & 0,957 & 2 & 75 & 1,692 & 0,191 & 0,043 \\
\hline $\begin{array}{l}\text { Forma } \\
\text { Gramatical }\end{array}$ & 0,751 & 2 & 75 & 12,465 & 0,00 & 0,249 \\
\hline $\begin{array}{l}\text { Forma } \\
\text { Gramatical } \\
* \text { Realce }\end{array}$ & 0,986 & 2 & 75 & 0,534 & 0,588 & 0,014 \\
\hline
\end{tabular}


EFECTOS DEL REALCE TEXTUAL Y LA FORMA GRAMATICAL SOBRE LA COMPRENSIÓN Y EL PROCESAMIENTO

José Franco (pp. 30-53)

Los resultados del Análisis Factorial Múltiple de Varianza permitieron posteriormente efectuar dos análisis de varianza simple, uno para la comprensión y otro para el procesamiento con el fin de verificar cuál de las dos variables dependientes fue afectada en mayor grado por la forma gramatical

\section{La Forma Gramatical y la Comprensión}

El Análisis de Varianza Simple para la Comprensión indicó que la forma gramatical tuvo efectos estadísticamente significativos sobre la comprensión. Los resultados son mostrados en la tabla 2.

Tabla 2. Análisis de Varianza Simple para la Comprensión.

\begin{tabular}{lcccccc}
\hline Fuente & $\begin{array}{c}\text { Suma de los } \\
\text { Cuadrados }\end{array}$ & $\begin{array}{c}\text { Grados de } \\
\text { Libertad }\end{array}$ & $\begin{array}{c}\text { Medias } \\
\text { Cuadráticas }\end{array}$ & F & p & *" \\
\hline Realce & 1599,292 & 1 & 1599,292 & 2,594 & 0,111 & 0,033 \\
\hline $\begin{array}{l}\text { Forma } \\
\text { Gramatical }\end{array}$ & 10518,168 & 1 & 10518,168 & 17,063 & 0,000 & 0,183 \\
\hline $\begin{array}{l}\text { Forma } \\
\text { Gramatical } \\
* \text { Realce }\end{array}$ & 363,173 & 1 & 363,173 & 0,589 & 0,445 & 0,008 \\
\hline Error & 46848,437 & 76 & 616,427 & & & \\
\hline
\end{tabular}

Las medias y desviaciones estándar del Análisis de Varianza Simple para la Comprensión indicaron que la forma pasiva $(\mathrm{M}=43,45)$ fue comprendida en mayor grado que el presente perfecto $(M=20,51)$ independientemente del realce textual o su interacción con la forma gramatical. Los resultados son mostrados en la tabla 3.

Tabla 3. Medias y desviaciones estándar del Análisis de Varianza Simple para la Comprensión.

\begin{tabular}{clccc}
\hline $\begin{array}{c}\text { Forma } \\
\text { Gramatical }\end{array}$ & Realce Textual & Media & Desviación Estándar & N \\
\hline \multirow{3}{*}{ Forma Pasiva } & Realzado & 41,11 & 25,81 & 21 \\
\cline { 2 - 5 } & No Realzado & 45,90 & 30,99 & 20 \\
\cline { 2 - 5 } & Total & 43,45 & 28,20 & 41 \\
\hline & Realzado & 13,68 & 14,60 & 19 \\
\hline
\end{tabular}


EFECTOS DEL REALCE TEXTUAL Y LA FORMA GRAMATICAL SOBRE LA COMPRENSIÓN Y EL PROCESAMIENTO

José Franco (pp. 30-53)

\begin{tabular}{lllll}
\hline Presente Perfecto & No Realzado & 27,00 & 24,51 & 20 \\
\cline { 2 - 5 } & Total & 20,51 & 21,14 & 39 \\
\hline \multirow{3}{*}{ Total } & Realzado & 28,08 & 25,15 & 40 \\
\cline { 2 - 5 } & No Realzado & 36,45 & 29,19 & 40 \\
\cline { 2 - 5 } & Total & 32,26 & 27,40 & 80 \\
\hline
\end{tabular}

\section{La Forma Gramatical y el Procesamiento}

El Análisis de Varianza Simple para el procesamiento indicó que la forma gramatical tuvo un efecto estadísticamente significativo sobre el procesamiento. Los resultados son mostrados en la tabla 4.

Tabla 4. Análisis de Varianza Simple para el Procesamiento.

\begin{tabular}{lcccccc}
\hline Fuente & $\begin{array}{c}\text { Suma de los } \\
\text { Cuadrados }\end{array}$ & $\begin{array}{c}\text { Grados de } \\
\text { Libertad }\end{array}$ & $\begin{array}{c}\text { Medias } \\
\text { Cuadráticas }\end{array}$ & F & p & *" \\
\hline Realce & 124,577 & 1 & 124,577 & 0,088 & 0,767 & 0,001 \\
\hline $\begin{array}{l}\text { Forma } \\
\text { Gramatical }\end{array}$ & 2203,401 & 1 & 2203,401 & 1,564 & 0,215 & 0,020 \\
\hline $\begin{array}{l}\text { Forma } \\
\text { Gramatical } \\
* \text { Realce }\end{array}$ & 216,612 & 1 & 216,612 & 0,154 & 0,696 & 0,002 \\
\hline Error & 357432,250 & 80 & 1408,689 & & & \\
\hline
\end{tabular}

Las medias y desviaciones estándar del Análisis de Varianza Simple para el procesamiento indicaron que el presente perfecto $(\mathrm{M}=61,03)$ tiende a ser procesado en mayor grado que la forma pasiva $(\mathrm{M}=50,53)$ independientemente del realce textual o su interacción con la forma gramatical. Los resultados son mostrados en la tabla 5.

Tabla 5. Medias y desviaciones estándar del Análisis de Varianza Simple para el Procesamiento

\begin{tabular}{clccc}
\hline $\begin{array}{c}\text { Forma } \\
\text { Gramatical }\end{array}$ & Realce Textual & Media & Desviación Estándar & N \\
\hline \multirow{3}{*}{ Forma Pasiva } & Realzado & 50,19 & 38,74 & 21 \\
\cline { 2 - 5 } & No Realzado & 50,90 & 35,68 & 20 \\
\cline { 2 - 5 } & Total & 50,53 & 36,81 & 41 \\
\hline & Realzado & 64,05 & 39,54 & 19 \\
\hline
\end{tabular}


EFECTOS DEL REALCE TEXTUAL Y LA FORMA GRAMATICAL SOBRE LA COMPRENSIÓN Y EL PROCESAMIENTO

José Franco (pp. 30-53)

\begin{tabular}{lllll}
\hline Presente Perfecto & No Realzado & 58,17 & 36,05 & 20 \\
\cline { 2 - 5 } & Total & 61,03 & 37,40 & 39 \\
\hline \multirow{3}{*}{ Total } & Realzado & 56,77 & 39,25 & 40 \\
\cline { 2 - 5 } & No Realzado & 54,54 & 35,59 & 40 \\
\cline { 2 - 5 } & Total & 55,65 & 37,24 & 80 \\
\hline
\end{tabular}

El efecto estadísticamente significativo del aspecto gramatical sobre la comprensión indicó que, independientemente del realce textual, la forma pasiva resultó más beneficiosa en términos de comprensión que el presente perfecto. Asimismo, la tendencia estadísticamente significativa del aspecto gramatical sobre el procesamiento indicó que, independientemente del realce textual, el presente perfecto resultó más beneficioso en términos de procesamiento que la forma pasiva. Este hallazgo es mostrado en la Figura 2.

\section{DISCUSIÓN DE LOS RESULTADOS Y RECOMENDACIONES}

La presente investigación tuvo tres propósitos fundamentales. El primero, establecer las posibles diferencias entre los estudiantes de las dos instituciones seleccionadas, en términos de comprensión y procesamiento. El segundo, examinar los posibles efectos del realce textual sobre la comprensión y el procesamiento. El tercer propósito fue verificar los posibles efectos de las formas gramaticales sobre la comprensión y el procesamiento.

En relación al primer propósito, los resultados obtenidos de la prueba $t$ mostraron que no hubo diferencias interinstitucionales en términos de comprensión, $(\mathrm{p}=0,78)$ ni de procesamiento $(\mathrm{p}=0,90)$ de las formas gramaticales, razón por la cual, ambas instituciones fueron tomadas como una sola muestra. Esto pudiera deberse primordialmente a que los programas de enseñanza de inglés como Lengua Extranjera de la Universidad del Zulia y del Instituto Pedagógico Experimental Libertador son similares en cuanto a contenido.

Cabe destacar que en anteriores investigaciones en las que se incluye la participación de estudiantes de instituciones diferentes (Wong, ob. cit; Lee, ob. cit y Simard, ob. cit), no se establecen comparaciones entre las mismas en lo que respecta a comprensión, procesamiento o alguna otra variable. Esto pudiera deberse a que las muestras resultaran homogéneas y por ende fueran tratadas como una sola muestra, siendo éste un aspecto que vendría a ratificar los hallazgos descritos anteriormente referentes a la muestra. 
Con respecto al segundo propósito, los resultados revelaron que el realce textual no tuvo un impacto significativo en la comprensión y/o el procesamiento de los participantes al ser comparados con aquellos que no fueron referidos a dicha técnica. En otras palabras, el realce de la forma pasiva y el presente perfecto a través del uso de las letras mayúsculas, no fue efectivo para atraer la atención de los estudiantes hacia dichas formas en cuanto a la comprensión y/o procesamiento de textos.

Los resultados del Análisis Factorial Múltiple de Varianza mostraron que independientemente del realce de las formas gramaticales, estas fueron comprendidas o procesadas. Resultados éstos, aparentemente contradictorios con los reportados en otras investigaciones (Shook, 1994 citado en Leow, ob. cit; Jourdenais y otros, 1995 citado en Leow, ob. cit; Lee, ob. cit; De Santis, ob. cit y Simard, ob. cit), las cuales mostraron los efectos significativos del realce textual sobre el procesamiento de ciertas formas gramaticales.

En lo concerniente a los efectos de la forma gramatical sobre la comprensión y el procesamiento, se observaron resultados bastante interesantes. Tomando en cuenta los planteamientos de Han, Park y Combs (ob. cit), Leow y otros (ob. cit) y VanPatten (1996; citado en Wong, ob. cit), las formas gramaticales empleadas en el presente estudio, se pueden caracterizar como formas de impacto visual considerable. Los resultados del Análisis Factorial de Varianza para la comprensión y el procesamiento mostraron que la forma pasiva fue más beneficiosa para la comprensión, mientras que el presente perfecto resultó más favorable para el procesamiento. Cabe destacar que aun cuando estas formas gramaticales ya poseían un impacto visual inherente (Wong, ob. cit) y considerando que las mismas fueron realzadas con letras mayúsculas, los resultados no fueron los esperados.

Tomando en cuenta la composición morfológica de las formas empleadas en el presente estudio, podemos destacar que ambas están compuestas por dos morfemas (un verbo auxiliar y un verbo en participio pasado) y que a la vez uno de éstos es común para ellas (el participio pasado). Partiendo de esta idea, es posible que los resultados obtenidos del Análisis Factorial de Varianza para la comprensión y el procesamiento se deban a dos factores claves:

1) El verbo auxiliar empleado en cada forma gramatical. 
2) La naturaleza de las pruebas de comprensión y procesamiento.

Haciendo referencia al primer factor, es evidente que el verbo auxiliar to be de la forma pasiva es uno de los aspectos primordiales de la enseñanza de una SL o LE que se presentan en el aula de clase, en tal sentido es muy probable que el mismo hubiera sido presentado a los participantes previamente a la aplicación de los instrumentos, incidiendo considerablemente en los resultados antes planteados. Del verbo auxiliar to have del presente perfecto, sin embargo, se podría decir que aunque es un elemento igualmente primordial al anterior, cabría considerar si fue presentado en igual grado que el verbo to be.

En relación a la naturaleza de las pruebas de comprensión y procesamiento, éstas presentan unas diferencias bien marcadas. La prueba de comprensión (Leow, ob. cit) consistió en responder en español algunas preguntas relacionadas directamente con el texto, mientras que la prueba de procesamiento (Leow, ob. cit) consistió en elegir la forma gramatical correcta de cuatro opciones que fueron presentadas en 11 ítems distintos, dicha elección fue hecha de acuerdo a lo planteado en el texto.

Integrando estos aspectos, podría plantearse que los participantes estuviesen familiarizados en gran medida con el verbo auxiliar to be utilizado en la forma pasiva (cuya traducción al español es menos compleja que la del verbo to have), aunado a una prueba de comprensión cuyas preguntas debían ser respondidas en el idioma nativo de los participantes, pudiera haber afectado considerablemente la comprensión.

En el caso de la prueba de procesamiento, donde se debía elegir la opción correcta de la forma gramatical de cuatro que se presentaban, habría que reflexionar acerca de las variaciones que el verbo auxiliar to be de la forma pasiva y el verbo auxiliar to have del presente perfecto presentan en la conjugación del presente. Por un lado el verbo to be presenta cuatro formas: be, am, are e is. El verbo to have, por otro lado, presenta solamente dos formas diferentes: have y has. En resumen, en la prueba de procesamiento, el verbo to be pudo haber presentado más dificultad para los estudiantes en vista de las variaciones presentadas, mientras que el verbo to have que sólo presentó dos variaciones pudo haber presentado menos dificultad, afectando considerablemente el procesamiento. 
El hecho de desconocer la programación desarrollada por los profesores encargados de los cursos de los participantes limita una explicación más detallada de lo expresado anteriormente en otros capítulos, ya que al momento de la aplicación de las pruebas se pidió información acerca de las formas gramaticales que se aplicarían en el presente estudio, mas no de sus verbos auxiliares (to be y to have). En tal sentido, los hallazgos de este estudio podrían servir para explicar, por lo menos en parte, los efectos que la forma gramatical tiene sobre la comprensión y el procesamiento.

Finalmente, aunque lo planteado anteriormente pudiera de cierta forma resultar especulativo, es de suma importancia establecer las diferencias entre comprensión y procesamiento. Pulido (2007) indica que el nivel de procesamiento está en gran parte determinado por el nivel de comprensión del input, sin embargo, considerando esta afirmación, valdría la pena preguntarse ¿Por qué una forma gramatical (la forma pasiva) a pesar de ser comprendida, no es procesada en el mismo grado?

Futuras investigaciones deberían indagar más a fondo lo referente a la naturaleza de las formas gramaticales. Asimismo, los profesores de inglés deben diseñar actividades de clase y tareas que se adapten al grado de dificultad de tales formas gramaticales para promover su procesamiento más efectivamente.

\section{REFERENCIAS}

Aghajani, A. y Rahimy (2013). The Impact of Input Enhancement Type on Iranian EFL Learner's Knowledge of English Tenses across Genders. Asian Journal of Social Sciences \& Humanities, 2-1, 153-164.

Ayiewbey, S. (2013). An Investigation to the Effects of Textual Enhancement on the Learning of English Past Tense in the Context of Iranian Learners. Technical Journal of Engineering and Applied Sciences, 3-13, 1217-1222.

Barcroft, J. (2003). Distinctiveness and bidirectional effects in input enhancement for vocabulary learning. Applied Language Learning, 13, 47-73.

Dalili, M., Ketabi, S., Kassaiam, Z. y Rasekh, A. (2011). The Effect of Textual Enhancement on System Learning of English Dative Alternation. World Applied Science Journal (Innovation and Pedagogy for Lifelong Learning), 15, 63-69

De Santis, P. (2008). Text enhancement and the acquisition of english verbal inflection $-\mathrm{s}$ by L1 haitian creole speakers. Applied Language Learning, 18, 27-49. 
Han, Z., Park, E. y Comb, C. (2008). Textual enhancement of input: issues and possibilities. Applied Linguistics, 29/4, 597-618.

Hernández, R., Fernández, C. y Baptista, L. (1998). Metodología de la Investigación. México: Mc Graw Hill.

Izumi, S. (2002). Output, input enhancement, and the noticing hypothesis: an experimental study of SLA relativization. Studies in Second Language Acquisition, 24, 541-577.

Jabbarpoor, S. y Adbollahzadeh, E. (2013). Textual Enhancement across Linguistic Structures: EFL Learners' Acquisition of English Forms. Journal of Language and Translation, 3, 2, 69-78.

Jensen, E. y Vinther, T. (2003). Exact repetition as input enhancement in second language acquisition. Language Learning, 53:3, 373-428.

Lee, S. (2007). Effects of textual enhancement and topic familiarity on korean EFL students' reading comprehension and learning of passive form. Language Learning, 57, 87-118.

Leow, R. (1993). To simplify or not simplify: a look at intake. Studies in Second Language Acquisition, 15: 333-355.

Leow, R. (1997). The effects of input enhancement and text length on adult L2 reader's comprehension and intake in second language acquisition. Applied Language Learning, 8, 151-182.

Leow, R. (2001). Do learners notice enhanced forms while interacting with the L2? An online and offline study of the role of written input enhancement in L2 reading. Hispania, 84, 496-509.

Leow, R., Egi, T., Nuevo, A., \& Tsai, Y. (2003). The roles of textual enhancement and type of linguistic item in adult L2 learner's comprehension and intake. Applied Language Learning, 13, 93-108.

Long, M. H. (1991). Focus on form: A design feature in language teaching methodology. In K. de Bot, R. Ginsberg and C. Kramsch (Eds.), Foreign Language Research in Crosscultural Perspective, (pp: 39-52). Amsterdam: John Benjamins.

Nahavandi, N. y Mukundan, J. (2013). The Impact of Textual Input Enhancement and Explicit Rule Presentation on Iranian Elementary EFL Learner's Intake of Simple Past Tense. English Language Teaching, 6/01, 92-102.

Poole, A. (2005). Focus on form instruction: foundations, applications, and criticisms. The Reading Matrix. 5,1, 47, 56.

Pulido, D. (2007). The relation between text comprehension and second language incidental vocabulary acquisition: a matter of topic familiarity? Language Learning, 57, 1, 155199.

Raimes, A. (1991). Out of the woods: Emerging traditions in the teaching of writing. Tesol Quaterly. 25,3. 
Richards, J, Platt, J., Weber H. (1985). Longman Dictionary of Applied Linguistics. Longman Group. England.

Robinson, P. (1995). Attention, memory, and the noticing hypothesis. Language Learning, 45, 283-331.

Sarkhosh, M. (2012). Different Textual Enhancement Formats and the Intake of English Past Simple Tense. International Journal of Linguistics, 4/2, 459-474.

Sharwood Smith, M. (1994). Second language learning: theoretical foundations. Longman, New York.

Simard, D. (2009). Differential effects of textual enhancement formats on intake. System, $37,124-135$.

Wolff, D. (1987). Some assumptions about second language text comprehension. Studies in Second Language Acquisition, 9, 307-326.

Wong, W. (2003). Textual enhancement and simplified input: effects on L2 comprehension and acquisition of non-meaningful grammatical form. Applied Language Learning, 13, $17-45$. 